DOI: $10.3901 / J M E .2014 .23 .112$

\title{
含氮硼蓖麻油聚氧乙烯醚水溶液的摩擦学特性
}

\author{
何建国 ${ }^{1,2}$ 张朝辉 $^{1}$ 刘思思 ${ }^{1}$ 周 杰 $^{1}$ 凡炼文 ${ }^{1}$ \\ (1. 北京交通大学机械与电子控制工程学院 北京 100044 ; \\ 2. 中国科学院光电研究院 北京 100094)
}

\begin{abstract}
摘要: 聚醚水溶液是水基润滑液的重要研究方向之一。为改善水的摩擦学性能, 选取蓄麻油聚氧乙烯醚系列中的 EL-60(Cremophor EL-60)和三乙醇胺硼酸酯(Boric acid triethanolamine, BN)作为添加剂, 并对相关溶液进行对比分析研究, 同 时对两种物质对水溶液摩擦学特性的影响进行了一定论述。EL-60 是一种性能良好的表面活性剂, 能够充分吸附在金属表面, 形成牢固的润滑分子层; BN 具有良好的极压性能和防锈性能。配置 EL-60 水溶液及两种物质混合质量比为 1:1 的 BN-EL-60 水溶液, 利用 MRS-10A 型四球试验机对 EL-60 水溶液以及 BN-EL-60 水溶液进行减摩抗磨性能试验与极压性能试验、防锈 特性研究等。结果表明 BN-EL-60 溶液在高负荷情况下具有一定性能优势, 而低负荷情况 EL-60 单质溶液则相对优秀; 较低 浓度的溶液就已经具备较好的减摩抗磨综合性能; 加入 BN 之后溶液的摩擦因数随时间的变化波动增大, 溶液摩擦学性能稳 定性相对变差; 另外, BN 的引入能够大大提升溶液的极压性能和防锈性能。BN 与 EL-60 的协同效应并非是单纯协同增效, 其性能是随工作负荷的变化而改变的, 总体而言, EL-60 与 BN-EL-60 是一类具有良好应用前景的水溶液润滑添加剂。
\end{abstract}

关键词: 水基润滑液; 减摩抗磨; 极压防锈; 蓄麻油聚氧乙烯醚; 三乙醇胺硼酸酯

中图分类号: TH117

\section{Tribological Properties of Cremophor EL Aqueous Solutions Containing Nitrogen and Boron}

\author{
HE Jianguo ${ }^{1,2}$ ZHANG Chaohui ${ }^{1} \quad$ LIU Sisi $^{1} \quad$ ZHOU Jie $^{1} \quad$ FAN Lianwen $^{1}$ \\ (1. School of Mechanical, Electronic and Control Engineering, Beijing Jiaotong University, Beijing 100044; \\ 2. Academy of Opto-Electronics, Chinese Academy of Sciences, Beijing 100094)
}

\begin{abstract}
Polyether aqueous solution is one of the important research in water-based lubricant. Cremophor EL-60 is an excellent surfactant, it can fully adsorb on the metal surface, and forming firm lubricant molecular layer. Boric acid triethanolamine (BN) has excellent extreme pressure properties and anti-rust properties. Mixing the two additives in mass ratio of 1:1, there is the BN-EL-60 water-based lubricant. Anti-friction and anti-wear performances of EL-60 aqueous solutions and BN-EL-60 aqueous solutions is studied by MRS-10 four ball tester, so as to the performances of extreme pressure, anti-rust, etc. It is showed that the BN-EL-60 aqueous solutions have a better performances at high load and high rotating speed, and at normal conditions EL-60 aqueous solutions are better. The two series have a good anti-friction and anti-wear performances at low concentration; after bringing BN into the solutions the friction coefficient fluctuation becomes unstable, but it can also obviously improve the extreme pressure performances and anti-rust performances. The synergistic behavior of BN and EL-60 additives are not simply, its characteristics are changing with the working conditions. Overall, EL-60 and BN-EL-60 additives are kinds of water-soluble additives with good application prospects.

Key words: water-based lubricant; anti-friction and anti-wear; extreme pressure and anti-rust; cremophor EL; boric acid triethanolamine
\end{abstract}

\section{0 前言}

聚醚(一种嵌段复合物)又称聚乙二醇醚 ${ }^{[1]}$, 是表 面活性剂产品中的佼佼者, 在金属加工液产业中具 有重要的地位, 也是水基润滑液领域研究的重要方
向之一。由于它的表面活性, 具备强劲的界面润湿 能力和吸附力, 因而能够在金属表面形成牢固的润 滑吸附膜, 能够起到抗磨的作用。聚醚的黏度大于 一般的油基基础油, 而其低浓度的水溶液黏度较低, 运行过程中的黏度较为稳定, 在工作时剪切力处于 较低的水平, 这对减摩性能有着重要作用 ${ }^{[2-3]}$ 。聚醚 的黏度特性随着聚醚相对分子量的增加而增大, 较

20131213 收到初稿, 20140701 收到修改稿 
高的黏度有助于形成更加稳定的物理吸附膜和流体 润滑膜, 能够在某些情况下提高润滑系统的综合性 能。文献[4]在聚氧乙烯基醚水基润滑液摩擦学特性 研究中详细分析了四种聚氧乙烯基醚在水溶液中的 摩擦学性能, 并从动力黏度、成膜性能、减摩抗磨 性能几个方面进行了综合分析, 证明四种聚醚中蓖 麻油聚氧乙烯醚 EL-40 在水溶液中的减摩抗磨性能 较为优秀、成膜能力较强, 是一种性能优秀的水基 润滑添加剂。

有机嗍酸酯是一类多功能添加剂 ${ }^{[4]}$ 。含嗍表面 活性剂的分子中一般具有 B-O 键, 且为非离子型, 沸点很高, 高温下稳定, 具有较好的抗菌性能, 是 一种无毒、无公害、易合成, 具有杀菌、防腐、抗 磨能力的性能优秀的新型表面活性剂, 并且近年来 的研究已经很好地解决了水解的问题 ${ }^{[5,7-12]}$, 避免了 其水解现象对水基润滑液性能负面影响的出现。胡 志孟 ${ }^{[6]}$ 在一种新型的水溶性有机硼化合物润滑和防 锈添加剂中提到一种性能优秀的水基添加剂, 其 $\mathrm{P}_{\mathrm{B}}$ 值为 $784 \mathrm{~N}$, 在 $196 \mathrm{~N}$ 的四球机长磨试验下, 钢球 的磨斑直径为 $0.41 \mathrm{~mm}$, 同时还进行了防锈、防腐 蚀、大白鼠急性毒性试验等, 充分证明了含氮嗍有 机化合物添加剂是一种具有发展前途的优秀添 加剂。

为提高水的摩擦学性能, 需要解决水的低黏度 成膜难、界面润湿能力相对较差、容易使浸入其中 的金属发生锈蚀等几类问题。本文总结了聚醚类水 基润滑液及含硼添加剂研究领域的相关成果, 选取 聚氧乙烯醚系列中相对分子量较高的菎麻油聚醚与 不水解的含氮硼化合物作为水基润滑添加剂, 以期 所配置的溶液具备二者优点, 并出现协同增效情况。 由于两物质不会发生化学反应, 性质相对稳定, 不 易出现竞争吸附, 在水溶液中能够发挥各自的性能 优势。本文所得的研究结果能够一定程度揭示两种 物质对水溶液摩擦学特性的影响, 帮助形成一类具 备易成膜、膜性能优秀、黏度适中、极压性能良好, 抗菌、绿色环保的新型水基润滑液。

\section{1 试验设备和试验方案}

\section{1 添加剂的选择}

EL-60 是䔣麻油聚氧乙烯醚系列中相对分子量 较高的表面活性剂物质, 分子极性强, 分子链长, 能够充分吸附在摩擦副表面, 形成牢固的润滑分子 层, 为摩擦副提供良好的润滑性能。考虑到 EL-60 是一种有容易被分解处理的机高分子聚合物, 符合 环境友好型的要求, 同时它也能用于日化和制药领
域, 是一种环境兼容性和人体兼容性极佳的表面活 性剂, 能够有效解决水基润滑液的处理与日常管理 问题, 满足人们对绿色环保的需求。

$\mathrm{BN}$, 即三乙醇胺硼酸酯, 是含氮硼酸酯中一种 热稳定性良好, 不发生水解的嗍酸酯类添加剂, 它 含有嗍元素, 在极端负荷下能够与表面物质生成㸴 化物, 形成致密的氧化保护膜或是硼的渗透层, 起 到增强润滑和保护表面的作用 ${ }^{[9,13-14]}$; 由于它的碱 度较高, 因而是一种良好的 $\mathrm{pH}$ 值稳定剂, 特别是 对于以水为基础的水基润滑液来说，它是一类性能 良好的极压防锈添加剂。

添加剂选取菎麻油聚氧乙烯醚 EL-60 与三乙醇 胺硼酸酯(BN)作为添加剂, 配置了 EL-60 单质溶液 与 BN-EL-60 溶液, 浓度共八种, 分别为 $0.5 \% 、 1 \%$ 、 $5 \% 、 10 \% 、 15 \% 、 20 \% 、 25 \% 、 30 \%$ 。采用去离子水 溶解溶质, 静置 $20 \mathrm{~min}$ 后, 利用双向磁力摚拌机摚 拌 $30 \mathrm{~min}$, 静置 $2 \mathrm{~h}$, 观察溶液是否出现分层。在 所配置的溶液中, 由于添加剂对水的溶解性均为易 溶, 因此溶解较快, 且溶解后不会出现分层, 浓度 越大, 溶解越慢。

\section{2 试验设备与试验方案}

试验设备选取由济南试金集团生产的 MRS-10A 型四球试验机对所配置的溶液进行减摩 抗磨性能与极压性能研究。

试验分四部分, 前三部分主要考察溶液的减摩 抗磨性能, 分别以浓度为变量、以载荷为变量、以 转速为变量, 从三方面考察溶液性能; 第四部分考 察溶液的极压性能。其中试验从以浓度为变量的系 列试验中选取 $1 \%$ 浓度的溶液作为后续试验的研究 对象。

\section{2 试验结果与讨论}

\section{1 两溶液减摩抗磨性能分析}

\subsection{1 浓度对减摩抗磨性能的影响}

该组试验的试验条件: 载荷 $200 \mathrm{~N}$, 转速 1435 $\mathrm{r} / \mathrm{min}$ ，时间 $30 \mathrm{~min}$, 浓度从 $0.5 \%$ 变化至 $30 \%$ 。

从图 1 和图 2 中的数据可以看出, 无论是 EL-60 溶液还是 BN-EL-60 溶液在都在较低的浓度下就已 经具备了较好的减摩抗磨性能, 两溶液的摩擦因数 均随浓度的增加而下降至最低点, 此后浓度继续升 高则溶液的黏度增加, 黏度所带来的内切应力的提 高使溶液宏观表现出摩擦因数增加。

如图 2 所示, 两溶液的磨斑直径曲线截然相反, EL-60 溶液磨斑直径随浓度的上升有一定下降, 但 下降幅度不大, 而 BN-EL-60 溶液的磨斑直径随浓 
度的上升而不断变大, 最终稳定在 $0.85 \mathrm{~mm}$ 左右。

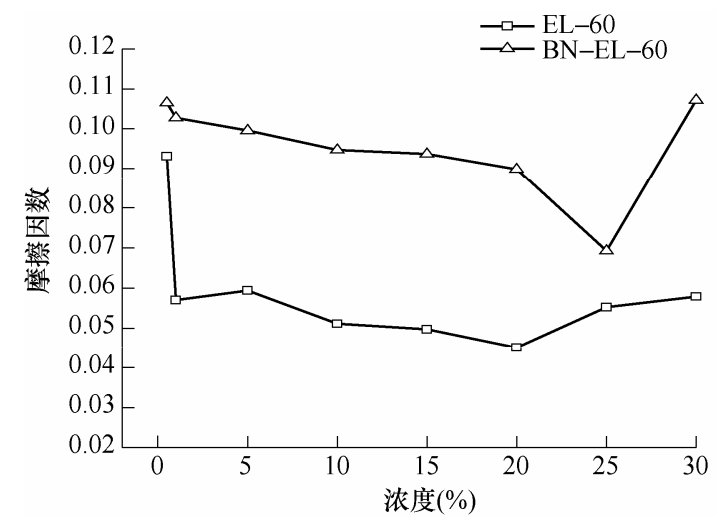

图 1 两种溶液不同浓度的摩擦因数曲线

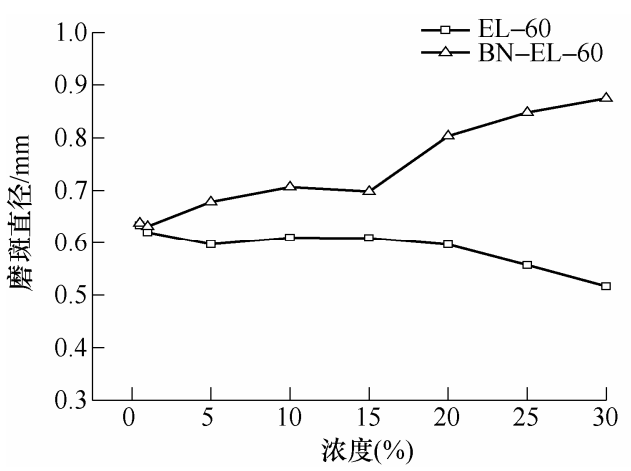

图 2 两种溶液不同浓度的磨斑直径曲线

因此, 图 1 与图 2 的结果可以说明低负荷情况 下, BN-EL-60 的减摩抗磨性能在所有浓度全面低 于 EL-60, 其中两溶液的摩擦因数随浓度变化均先 下降后升高, 而抗磨性能则变化单调, BN 组分越 大，抗磨性能越差。

由于 $\mathrm{BN}$ 水溶液的黏度很低, 含有 $\mathrm{BN}$ 的溶液 整体黏度下降, 加之 EL-60 组分减少、两种物质在 溶液中发生竞争吸附现象, 因而 BN-EL-60 溶液在 低负荷情况下, 无论是吸附膜还是流体膜强度均有 降低, BN-EL-60 溶液低负荷情况下性能相对较差。 考虑到水基润滑液大量应用于金属加工领域, 其特点为用量大、浓度低、成本低等, 因此选择 $1 \%$ 浓度的两溶液进行后续研究。

\subsection{2 载荷对减摩抗磨性能的影响}

该组试验的试验条件: 浓度 $1 \%$, 转速 1435 $\mathrm{r} / \mathrm{min}$, 时间 $30 \mathrm{~min}$, 载荷从 $200 \mathrm{~N}$ 变化至 $900 \mathrm{~N}$ 。

从图 3 可以看出, 低载荷下 $1 \% \mathrm{EL}-60$ 溶液摩擦 因数较 $1 \% \mathrm{BN}-\mathrm{EL}-60$ 低, 差距较大, 后者摩擦因数 较前者升高约 $80.2 \%$, 这与前述以浓度为变量时的 结果基本相符, 低载荷下 1\%EL-60 溶液性能优势明 显。随着载荷的增加, $1 \% \mathrm{EL}-60$ 溶液摩擦因数的变 化趋势为随载荷增加而增加, 而 $1 \% \mathrm{BN}-\mathrm{EL}-60$ 溶液 摩擦因数一直在下降, $400 \mathrm{~N}$ 左右时出现拐点,
$1 \% \mathrm{EL}-60$ 溶液摩擦因数由之前的上升, 开始转为下 降, 相对 $1 \%$ BN-EL-60 溶液摩擦因数的优势逐步减 小, 并且开始呈现相同的变化趋势。中等载荷之后 两者减摩性能基本相同, 趋势为逐步下降, 最终两 者都在 0.077 左右。整体而言, 两溶液减摩性能变 化趋势不同，低载荷下 $1 \% \mathrm{EL}-60$ 溶液强于 $1 \% B N-E L-60$ 溶液，中高载荷时 $1 \%$ BN-EL-60 溶液 性能提升, 并逐步超过 1\%EL-60 溶液最高达 5.3\%, 平均为 $2.4 \%$ 。

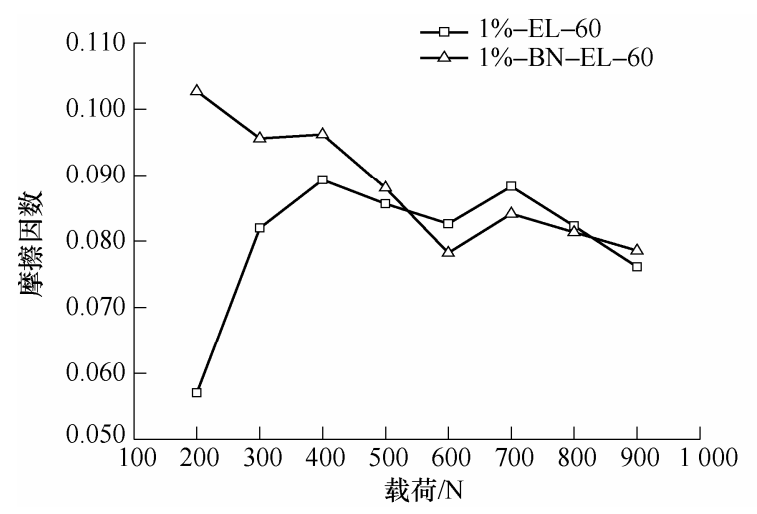

图 $31 \%$ 浓度两种溶液的摩擦因数随载荷的变化曲线

从图 4 可以看出, 两种溶液磨斑直径逐渐上升, 其中 $1 \% \mathrm{EL}-60$ 溶液上升较快, 而 $1 \% \mathrm{BN}-\mathrm{EL}-60$ 变化 平缓、性能稳定。中等载荷( $600 \mathrm{~N}$ 左右) 前 $1 \% \mathrm{EL}-60$ 溶液磨斑直径低于 $1 \% \mathrm{BN}-\mathrm{EL}-60$ 溶液, 而载荷继续 增加之后, $1 \% \mathrm{BN}-\mathrm{EL}-60$ 溶液性能优势开始体现, 最高优势达 $36.8 \%$ 。 $1 \% \mathrm{EL}-60$ 溶液磨斑直径在 0.548 5 1.121 $5 \mathrm{~mm}$ 之间, 1\%BN-EL- 60 磨斑直径 在 $0.6233 \sim 0.7083 \mathrm{~mm}$ 之间。整体而言, 中等载荷 $(600 \mathrm{~N}$ 左右)以上的平均磨斑直径, $1 \% \mathrm{BN}-\mathrm{EL}-60$ 溶 液抗磨性能较 $1 \% \mathrm{EL}-60$ 溶液提升近 $17.85 \%$ 。

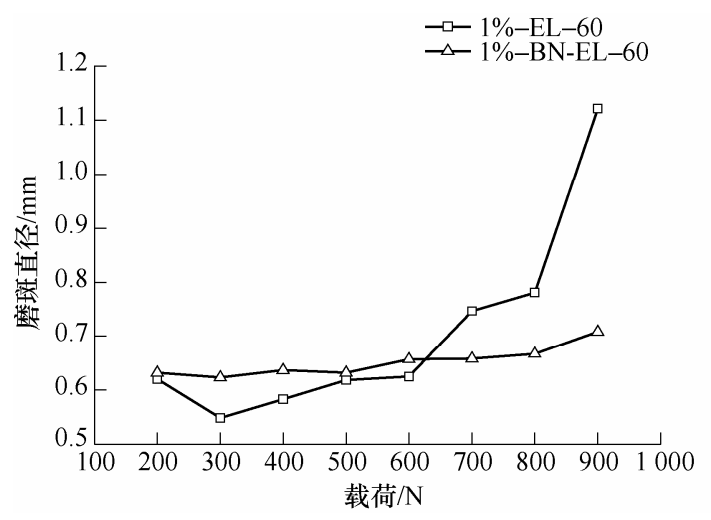

图 $4 \quad 1 \%$ 浓度两种溶液的磨斑直径随载荷的变化曲线

综合分析图 3 与图 4, 可以得到以下结论, $1 \%$ BN-EL-60 溶液在低载荷下摩擦磨损性能相对较 差, 在中高载荷下混合物中的 $\mathrm{BN}$ 开始发挥极压剂 的作用, 优化溶液的摩擦磨损性能。 


\subsection{3 转速对减摩抗磨性能的影响}

该组试验的试验条件：载荷 $200 \mathrm{~N}$, 浓度 $1 \%$, 时间 $30 \mathrm{~min}$, 主轴转速从 $835 \mathrm{~min}$ 变化至 $1840 \mathrm{r} / \mathrm{min}$ 。

从图 5 中曲线可以看出, $1 \% \mathrm{EL}-60$ 溶液减摩性 能较 $1 \% \mathrm{BN}-\mathrm{EL}-60$ 溶液优势明显, $1 \% \mathrm{EL}-60$ 溶液摩 擦因数在 $0.07902 \sim 0.09041$ 之间变化,而后者的摩 擦因数在 $0.10273 \sim 0.11620$ 之间变化。整体而言, $1 \% \mathrm{EL}-60$ 溶液减摩性能较 $1 \% \mathrm{BN}-\mathrm{EL}-60$ 溶液最大提 升 $47.05 \%$, 平均减摩性能提升 $29.64 \%$ 。在转速为 $1435 \mathrm{r} / \mathrm{min}$ 时出现拐点 $1 \% \mathrm{EL}-60$ 溶液由前面摩擦因 数上升的趋势变为下降, $1 \% \mathrm{BN}-\mathrm{EL}-60$ 溶液由前面 摩擦因数下降的趋势变为上升。

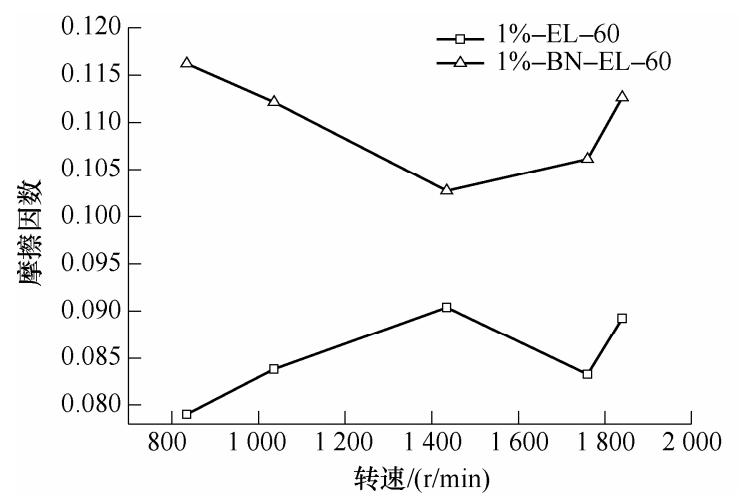

图 $51 \%$ 浓度两种溶液的摩擦因数随转速的变化曲线

从图 6 中可以看到, 两溶液磨斑直径变化趋势 完全不同, $1 \% \mathrm{EL}-60$ 溶液磨斑直径随转速的上升而 上升, 在 $1760 \mathrm{r} / \mathrm{min}$ 至 $1840 \mathrm{r} / \mathrm{min}$ 时, 摩擦因数出 现下降的情况; 而 $1 \% \mathrm{BN}-\mathrm{EL}-60$ 溶液磨斑直径波动 下降最终稳定在 $0.605 \mathrm{~mm}$ 。

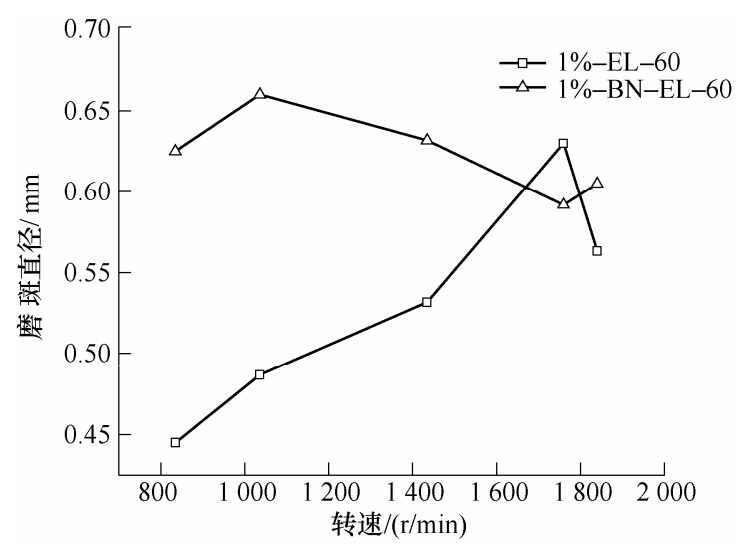

图 $61 \%$ 浓度两种溶液的磨斑直径随转速的变化曲线

$1 \% B N-E L-60$ 溶液磨斑直径在 $0.5917 \sim 0.6600$ $\mathrm{mm}$ 之间变化, 在低、中转速下抗磨性能较 1\%EL-60 劣势很大, 最大达 $40.45 \%$, 整体抗磨性能平均降低 $19.24 \%$ 。在中等转速之后两溶液的抗磨性能逐渐接 近, 在 $1600 \mathrm{r} / \mathrm{min}$ 左右, $1 \% \mathrm{BN}-\mathrm{EL}-60$ 溶液性能超 过 $1 \%$ EL-60 溶液, 超过最大达 $6.1 \%$, 说明
$1 \%$ BN-EL-60 溶液性能在中、高转速时较好。

\section{2 两溶液极压性能分析}

根据 GB/T12583-1998《润滑剂极压性能测定 法》 ${ }^{[15]}$, 利用四球试验机进行 EL-60 和 BN-EL-60 水溶液的极压性能测试。试验条件: 转速为 1760 $\mathrm{r} / \mathrm{min}$, 时间为 $10 \mathrm{~s}$, 结果归纳见表 1 。

表 1 极压性能试验结果

$\mathrm{N}$

\begin{tabular}{ccc}
\hline \multirow{2}{*}{ 不同浓度的溶液 } & \multicolumn{2}{c}{ 最大无咔咬负荷 } \\
\cline { 2 - 3 } & $\begin{array}{c}\text { EL-60 溶液 } \\
\mathrm{P}_{\mathrm{B}}(\mathrm{EL}-60)\end{array}$ & $\begin{array}{c}\text { BN-EL-60 溶液 } \\
\mathrm{P}_{\mathrm{B}}(\mathrm{BN}-\mathrm{EL}-60)\end{array}$ \\
\hline $1 \%$ & 543 & 849 \\
$10 \%$ & 834 & 1137 \\
\hline
\end{tabular}

结果显示, $\mathrm{BN}$ 的引入大大增加了聚醚水溶液 的极压性能, 在所体现的数据中可以看到 $1 \%$ 浓度 的 BN-EL-60 溶液较同浓度的 EL-60 溶液极压性能 提升 $56.4 \%, 10 \%$ 浓度的 BN-EL-60 溶液较同浓度的 EL-60 溶液极压性能提升 $36.3 \%$; 另外, 发现浓度 增加, 同样能够很好地提升水溶液自身的极压性能, 其中 EL-60 溶液 10\%浓度较 $1 \%$ 浓度极压性能提升 $53.6 \%$, 而 BN-EL-60 溶液 $10 \%$ 浓度较 $1 \%$ 浓度极压 性能提升 $33.9 \%$ 。

通过极压性能分析, 能够发现含有 $\mathrm{BN}$ 的溶液, 其极压性能十分优秀, $\mathrm{P}_{\mathrm{B}}$ 大于一般市售的水基极压 抗磨剂 ${ }^{[9]}$ 。由于两种物质的化学性质相对稳定, 在 溶液中不发生相互反应, 因此所提高的极压性能很 大程度上由 $\mathrm{BN}$ 所提供, 极压性能是考验溶液在极 端条件下的承载能力, 此时流体润滑膜以及物理化 学吸附膜已经被破坏, 极压剂其主要作用, 前文提 到 BN 在金属表面生成致密氧化反应膜或渗透膜, 可以证明 BN-EL-60 混合溶液中, 极端摩擦条件下, BN 作为极压抗磨剂体现出了它的优秀性能, 使该 型含氮硼聚醚溶液具备了优秀的极压性能, 拓展了 溶液的应用范围。

\section{3 防锈性能}

防锈性能试验选取减摩抗磨性能试验中所留 下的废液与钢球作为分析对象, 分别讨论了溶液在 低负荷、高载荷、高转速三种试验条件下的防锈性 能, 如图 7 9 所示。从中可以发现无论是 $0.5 \%$ 浓 度低负荷, 还是 $1.0 \%$ 浓度高载荷, 还是 $1.0 \%$ 浓度 高转速, 三种情况下含有 $\mathrm{BN}$ 溶质的溶液都具备相 对优秀的防锈性能。

其中, 从图 7 的四幅图片可以看出, 废液颜色 变浅, 另外钢球表面氧化物明显减少甚至消失; 从 图 8 的两幅图片则能够十分明显地发现, 在没有 BN 溶质的时候溶液呈现为赤铁矿的颜色, 说明溶液中 悬浮的金属切削颗粒物已经被氧化为 $\mathrm{Fe}_{2} \mathrm{O}_{3}$, 而引 
入 BN 后, 发现溶液颜色明显变浅虽然有一些浑浊, 但溶液中的漂浮物并没有严重氧化, 证明 BN 能够 极大地提高溶液的防锈性能; 从图 9 的两幅图片同 样能够发现, 引入 $\mathrm{BN}$ 的溶液其工作废液颜色较浅。

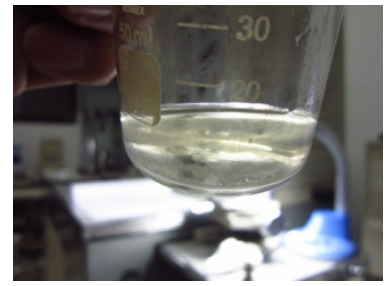

(a) $0.5 \% \mathrm{EL}-60$ 废液

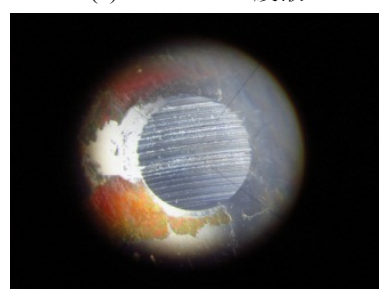

(c) $0.5 \%$ EL-60 钢球

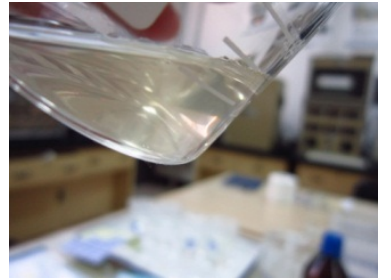

(b) $0.5 \% \mathrm{BN}-\mathrm{EL}-60$ 废液

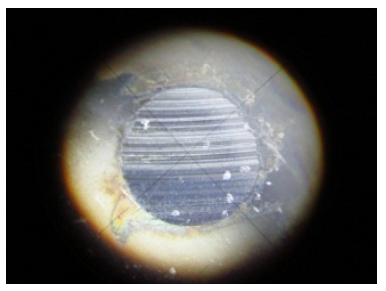

(d) $0.5 \% \mathrm{BN}-\mathrm{EL}-60$ 钢球
图 $70.5 \%$ 浓度两种溶液低负荷(载荷 $200 \mathrm{~N}$ 、转速 1435 $\mathrm{r} / \mathrm{min}$ )情况下的废液与钢球表面

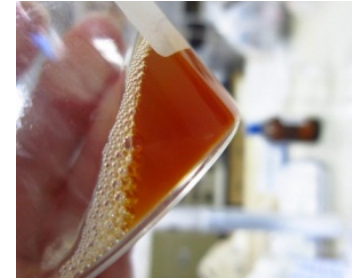

(a) $1.0 \% \mathrm{EL}-60$

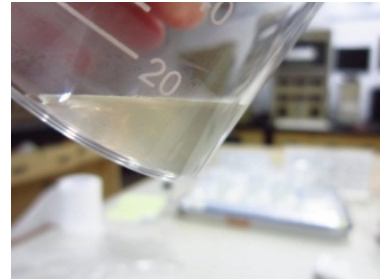

(b) $1.0 \% \mathrm{BN}-\mathrm{EL}-60$
图 $81 \%$ 浓度两种溶液高载荷(载荷 $900 \mathrm{~N}$ 、转速 $1435 \mathrm{r} / \mathrm{min}$ ) 情况下的工作废液

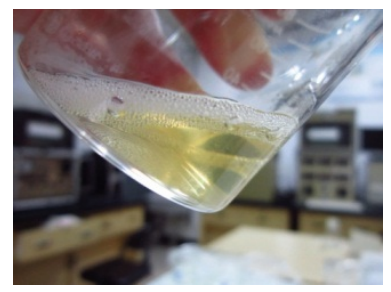

(a) $1.0 \% \mathrm{EL}-60$

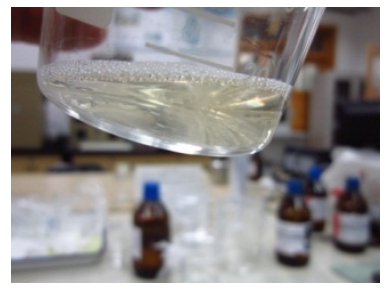

(b) $1.0 \% \mathrm{BN}-\mathrm{EL}-60$
图 $91.0 \%$ 浓度两种溶液高转速(载荷 $200 \mathrm{~N}$ 、转速 $1435 \mathrm{r} / \mathrm{min}$ ) 情况下的工作废液

分析以上试验结果, 较好地印证了前文所述的 关于 BN 防锈性能的内容, 从对比分析能够发现, 引入少量的 BN 到溶液中就能够起到很好的防锈性 能, 同时更高浓度的 BN-EL-60 能够具备更好的防 锈特性。

\section{4 稳定性能}

图 10 与图 11 为 EL-60 与 BN-EL-60 溶液的摩 擦因数随时间变化曲线, 从中发现极低浓度的润滑 液稳定性极差, 这是由于在低浓度时润滑液分子不
能很好地吸附在摩擦副表面, 同时溶液较低的黏度 使薄膜润滑中流体膜的性能很不稳定; 高浓度时虽 然润滑膜强度较大, 且摩擦副表面吸附达到饱和, 但稳定性同样处于一个较差的级别, 在高浓度情况 下主要的润滑因素应该是较强的流体膜和表面有序 吸附膜的作用, 然而作为水基润滑液, 在工作过程 中必定伴随着挥发和摩擦化学反应, 再考虑到聚醚 溶液的逆溶特性, 能够知道浓度越大, 挥发、摩擦 化学、逆溶等因素的基数越大, 因而其影响能力就 越大。伴随着挥发, 高浓度溶液的浓度和黏度变化 率将超过低浓度的溶液, 高浓度的聚醚溶液在逆溶 情况下所析出的物质也大大多于低浓度的溶液所析 出的物质。因此, 在实际考虑水基润滑液浓度的时 候, 浓度一定不能太低, 以避免溶液的润滑性能较 差, 同时也不能太高, 以避免溶液的稳定性较差。

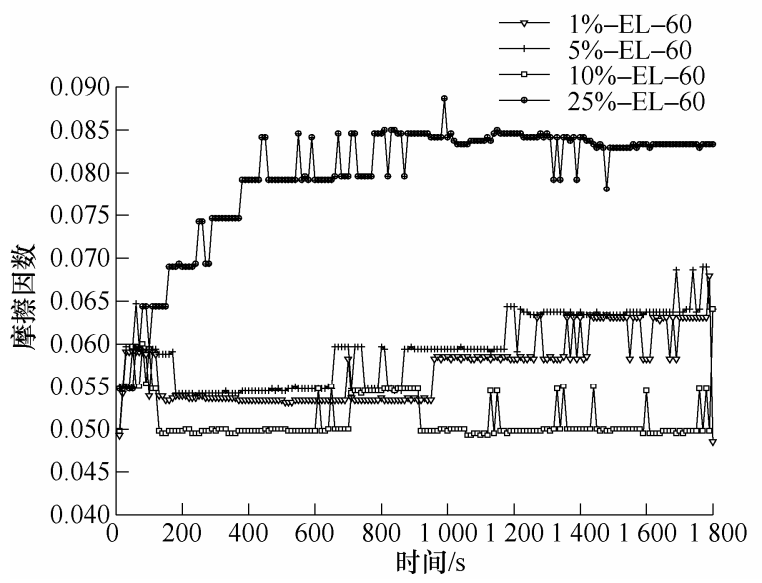

图 $10 \mathrm{EL}-60$ 系列溶液的摩擦因数随时间的变化曲线

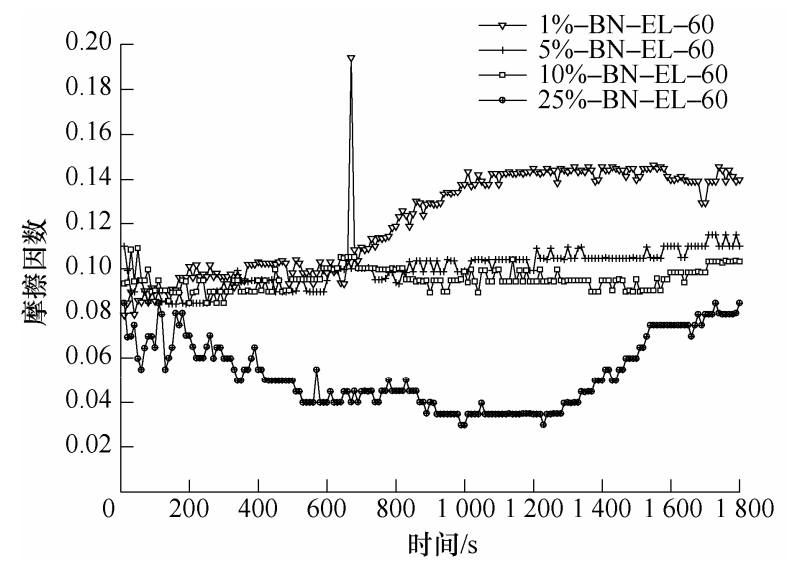

图 11 BN-EL-60 系列溶液的摩擦因数随时间的变化曲线

为详细分析两溶液的稳定特性, 对溶液的摩擦 因数求取标准差, 表征当次试验摩擦因数的波动特 性, 能够更好地分析溶液的稳定性能, 见图 12。

从图 12 中可以看出, BN-EL-60 溶液在所有浓 度下, 稳定性能均较 EL-60 溶液差, 两溶液在 $10 \%$ 浓度时稳定性能最接近, 且都为所有浓度中的最小 
值, 说明溶液在 $10 \%$ 浓度时稳定性能最好。

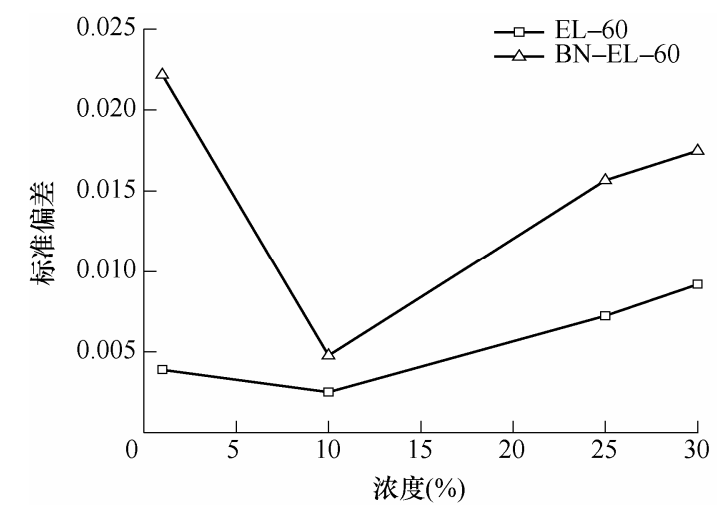

图 12 两种溶液摩擦因数标准差随浓度的变化曲线

含有 $\mathrm{BN}$ 的溶液, 在四球试验机试验过程中会 不断有晶体状物质析出, 观察发现是三乙醇胺硼酸 酯 $\mathrm{BN}$ 的晶体。考虑到 $\mathrm{BN}$ 晶体的不断出现以及其 在溶液中与溶液所形成的固液二相流的作用, 溶液 的整体性质必定是随 BN 晶体析出而变化的。至于 溶液变化的规律, 由于固液二相流的影响以及试验 溶液所处的复杂环境, 该规律无法预测, 也正因为 如此, 含有 $\mathrm{BN}$ 的溶液的稳定性较差且规律随机。

\section{3 结论}

(1) BN-EL-60 溶液在高负荷情况下的减摩抗磨 性能更强, 而在低负荷情况下 EL-60 溶液性能更好, 较低浓度的两溶液已经能够提供很好的减摩抗磨 性能。

(2) $\mathrm{BN}$ 的引入能够大大提升溶液极压性能与防 锈性能, 但其在溶液中的组分应适当, 以免润滑性 能损失太多。

(3) 含有 $\mathrm{BN}$ 的溶液会因为溶质析出, 稳定性 能下降, 两种溶液在 $10 \%$ 浓度时的稳定性能最好。

(4) 所选添加剂方案能够改善水的摩擦学特 性, 且具有一定防锈性能, 有望成为一类性能优秀 的环境友好型水基润滑液添加剂。

\section{参 考 文 献}

[1] 张康夫, 王余高, 屠伟刚, 等. 水基金属加工液 $[\mathrm{M}]$. 北 京: 化学工业出版社, 2007 .

ZHANG Kangfu, WANG Yugao, TU Weigang, et al. Water-based metalworking fluids[M]. Beijing: Chemical Industry Press, 2007.

[2] 李茂生. 水溶性聚醚在金属加工液中的应用 [J]. 合成 润滑材料，2003(30): 8-10.

LI Maosheng. Application of water-soluble PAGs in
Metalworking fluids[J]. Synthetic Lubricants, 2003(30): 8-10.

[3] 孙跃涛, 张朝辉, 刘思思. 月桂酸无规共聚醚水溶液减 摩抗磨性能试验研究[J]. 机械工程学报, 2011, 47(23): 125-129.

SUN Yuetao, ZHANG Chaohui, LIU Sisi. Study on Friction-reducing and anti-wear performances of lauric acid random copolyether aqueous solutions[J]. Journal of Mechanical Engineering, 2011, 47(23): 125-129.

[4] 刘俊铭. 聚氧乙烯基醚水基润滑液摩擦学特性研究[D]. 北京: 北京交通大学，2010.

LIU Junming. Study on tribological properties of water-based lubricants with polyethoxylate ether added[D]. Beijing: Beijing Jiaotong University, 2010.

[5] 胡晓兰, 梁国正. 嗍酸酯水解稳定性研究与应用 [J]. 材 料导报, 2002, 16(1): 58-60.

HU Xiaolan, LIANG Guozheng. Hydrolytic stability of borate ester and its application[J]. Materials Review, 2002, 16(1): 58-60.

[6] 胡志孟. 一种新型的水溶性有机硼化合物润滑和防锈 添加剂[J]. 材料保护, 2000, 33(4): 47-48.

HU Zhimeng. An investigation on water based organic boride for lubricating and rust resisting[J]. Materials Protection, 2000, 33(4): 47-48.

[7] 胡晓兰, 梁国正. 硼酸三乙醇胺酯的合成及表征 $[\mathrm{J}]$. 高 分子材料科学与工程, 2004, 20(2): 69-72.

HU Xiaolan, LIANG Guozheng. Synthesis and properties of reactive borate ester as coupling $[\mathrm{J}]$. Polymer Materials Science and Engineering, 2004，20(2): 69-72.

[8] WILLIAM E O. Lubricant compositions containing stable boric acid suspension: United States, 0015105[P]. 2011-01-20.

[9] 黄文轩. 润滑剂添加剂性质及应用[M]. 北京: 中国石 油化工出版社，2012.

HUANG Wenxuan. Lubricant additives properties and applications[M]. Beijing: China Petrochemical Press, 2012.

[10] ZHENG Zhi, SHEN Guangqiu, WAN Yong, et al. Synthesis hydrolytic stability and tribological properties of novel borate esters containing nitrogen as lubricant additives[J]. Wear, 1998(9): 135-144.

[11] YANG Honggang, ZHANG Jia, YE Meng. Research on the performance of an eco-friendly nitrogen organic boric acid ester antirust additive[J]. Advanced Materials Research, 2012, 399: 1348-1351.

[12] WANG Yonggang, CHU Xijie, HE Zhongyi, et al. 
Tribological performance of borate ester containing heterocyclic group and disulfides group[J]. Lubrication Engineering, 2010, 35(9): 36-39.

[13] LIEHPAO F O, ANDREW H G. Amine-containing hindered phenolic antioxidant lubricant additives and compositions thereof: United States, 5169547 [P]. 1992-12-08.

[14] ANDREW H G. Borated diol-phenol sulfide product and lubricant compositions containing same: United States, 4906390 [P]. 1990-03-06.

[15] 中华人民共和国国家质量监督检验检疫总局, 中国国 家标准化委员会. GB/T 12583-1998 润滑剂极压性能测 定法[S]. 北京: 中国标准出版社, 1995.
General Administration of Quality Supervision, Inspection and Quarantine of the People's Republic of China, Standardization Administration of the People's Republic of China. GB/T 12583-1998 lubricantsdetermination of extreme pressure properties-four ball method[S]. Beijing: China Standard Publishing House, 1995.

作者简介: 何建国, 男, 1988 年出生。主要研究方向为精密制造与摩 擦学。

E-mail: hejianguo@aoe.ac.cn

张朝辉(通信作者), 男, 1972 年出生, 博士, 教授, 博士研究生导师。 主要研究方向为精密制造与摩擦学。

E-mail: zhhzhang@bjtu.edu.cn 